\title{
Chronic Lymphocytic Leukaemia: Rare Involvement of the Central Nervous System
}

\author{
Carolina Amado ${ }^{1}$, Valter Duarte ${ }^{1}$, Mariana Silva Leal ${ }^{1}$, Margarida Cruz $^{1}$, Gisela Ferreira ${ }^{2}$ \\ ${ }^{1}$ Internal Medicine Department, Centro Hospitalar do Baixo Vouga, Aveiro, Portugal \\ ${ }^{2}$ Haematology Department, Centro Hospitalar do Baixo Vouga, Aveiro, Portugal
}

Received: 24/08/2021

Accepted: 05/10/2021

Published: 02/11/2021

\begin{abstract}
How to cite this article: Amado C, Duarte V, Silva Leal M, Cruz M, Ferreira G. Chronic lymphocytic leukaemia: rare involvement of the central nervous system. EJCRIM 2021;8: doi:10.12890/2021_002847.
\end{abstract}

Conflicts of Interests: The authors declare there are no competing interests.

This article is licensed under a Commons Attribution Non-Commercial 4.0 License

\section{ABSTRACT}

Involvement of the central nervous system, although uncommon, is one of the most frequent extramedullary manifestations of chronic lymphocytic leukaemia (CLL). Various conditions can lead to neurological symptoms in CLL patients and distinguishing between clinically significant CLL involvement of the CNS and other aetiologies can be challenging.

The authors report the case of a 90-year-old woman with a previous diagnosis of low-risk CLL who presented to the emergency room with altered mental status. After the most frequent causes were ruled out and considering the underlying disease, CNS infiltration by clonal B-cells was hypothesised and later confirmed. Treatment was initiated, but the patient died soon afterwards.

\section{LEARNING POINTS}

- Central nervous system involvement in chronic lymphocytic leukaemia (CLL) is rare and characterized by a heterogeneous clinical presentation.

- CNS localisation of CLL should be considered in patients with any neurological symptom, irrespective of the stage and activity of CLL.

- Brain imaging and cerebrospinal fluid examination should be performed.

\section{KEYWORDS}

Chronic lymphocytic leukaemia, central nervous system

\section{INTRODUCTION}

Chronic lymphocytic leukaemia (CLL) is the most common adult leukaemia in Western countries and is characterized by the proliferation and accumulation of small mature-appearing lymphocytes in the blood, bone marrow and lymphoid tissues ${ }^{[1]}$, the involvement of other locations being rare ${ }^{[2]}$. There are few reports on central nervous system (CNS) infiltration by CLL cells ${ }^{[3]}$, which can occur at any time during the natural history of the disease and is associated with lower overall survival ${ }^{[4]}$. Neurological complications arising from direct leukaemic involvement in the CNS are reported in only $1 \%$ of patients with $\mathrm{CLL}^{[5-7]}$. In contrast, post-mortem studies have demonstrated CNS involvement in up to $71 \%$ of patients with CLL, implying that CLL infiltration of the CNS is either underdiagnosed or frequently fails to manifest clinically ${ }^{[2]}$. Distinguishing whether neurological symptoms are due to CLL or other aetiologies can be challenging and may delay diagnosis and treatment ${ }^{[2,3,7]}$. 


\section{CASE DESCRIPTION}

A 90-year-old previously independent and normal-weight woman presented with a 1-day history of altered mental status (AMS). She had been diagnosed with CLL 3 years previously (Rai 0, Binet A) and was submitted to a watch-and-wait approach. She presented no other relevant medical history, such as new prescribed medications or supplements. There was no history of fever, or respiratory, urinary or gastrointestinal complaints.

On presentation, she was disoriented and agitated with an otherwise unremarkable neurological examination. Vital signs were within normal ranges. There were no other relevant findings on physical examination, namely, no adenomegalies, organomegalies, or auscultatory or skin alterations.

Blood work-up showed a haemoglobin of $10.6 \mathrm{mg} / \mathrm{dl}$ and a lymphocyte count of $34 \times 10^{\%} /$. . C-reactive protein, procalcitonin, thyroid function, serum electrolytes and vitamin B12 and folic acid were within normal ranges. HIV and VDRL serologies were negative. Urine analysis, a thoracic x-ray and abdominal ultrasound showed no signs of infection. No relevant abnormalities were observed on cerebral computed tomography. After ruling out the most frequent causes of disorientation and agitation, and considering the underlying disease, although apparently stable, CNS infiltration by CLL cells was hypothesised. A lumbar puncture was performed. Cerebrospinal fluid (CSF) was clear with normal glucose and protein levels, and negative bacterial culture. Immunophenotyping revealed the presence of lymphocytes positive for CD19, CD20weak, CD5, CD23, CD3 and CD200strong, confirming CNS infiltration by CLL cells. Based on the CSF findings along with the exclusion of other more common causes of acute confusional syndrome, the diagnosis of leptomeningeal CLL was established.

Palliative treatment with chlorambucil was commenced, but the patient died 1 month later.

\section{DISCUSSION}

The heterogeneity of clinical symptoms at presentation along with other more common differential diagnoses makes it a challenge to recognise CNS involvement in CLL ${ }^{[5]}$. This case highlights the importance of a graduated and personalized assessment of AMS in a 90-yearold patient with CLL. The patient underwent various tests to investigate five broad categories of common aetiologies of AMS ${ }^{[8]}$. Infections (mainly respiratory, skin and urinary), drug-related causes and metabolic or toxic encephalopathies such as hypoglycaemia, electrolyte abnormalities and hepatic or thyroid disorders, were all ruled out. Neurological causes such as stroke and cardiopulmonary diseases such as heart failure or hypoxia were also rejected. After excluding more common causes of AMS and as the patient had CLL, we suspected CNS infiltration by CLL cells.

AMS is a rare presentation of CLL, affecting only $15 \%$ of patients with CNS infiltration. Other symptoms to consider are those related to one or more cranial nerve palsies ${ }^{[6]}$. Leptomeningeal CLL is the most common CNS localisation. It often requires a high index of suspicion and is confirmed by neuroimaging and CSF analysis. Only the presence of malignant cells in the CSF is diagnostically conclusive ${ }^{[5,6]}$. This type of involvement in CLL patients is rare. The biological basis of lymphomatous involvement is not completely understood. One hypothesis suggests that the leptomeninges are seeded by systemically generated CLL cells, although the mechanisms of malignant transformation, the point of access to the CNS, and the origin of the cells remain unknown ${ }^{[9]}$.

Risk factors for CNS involvement in CLL have not been systematically explored ${ }^{[2]}$, although the presence of infectious agents can destabilize the blood-brain barrier ${ }^{[5]}$. Current knowledge suggests that CNS involvement is not related to disease stage, duration or progression ${ }^{[4]}$, or to a high-risk chromosomal abnormality, such as del11p or del111 $q^{[3]}$.

Of note, the patient did not show any symptoms that suggested Richter's syndrome (rapidly enlarged lymph nodes, fever and marked elevation of lactate dehydrogenase) [10].

Although current guidelines do not provide recommendations on the treatment of patients with CNS involvement in CLL, treatment with R-chlorambucil is usually preferred in frail patients with systemic symptoms ${ }^{[1]}$.

CNS involvement in CLL should be considered in patients with any neurological symptom, irrespective of the stage and activity of CLL. Both imaging and CSF analysis play a key role in the diagnosis and prompt initiation of appropriate treatment in these patients. 


\section{REFERENCES}

1. Eichhorst B, Robak T, Montserrat E, Ghia P, Niemann CU, Kater AP, et al. Chronic lymphocytic leukaemia: ESMO clinical practice guidelines for diagnosis, treatment, and follow-up. Ann Oncol 2020;32(1):23-33.

2. Strati P, Uhm Joon H, Kaufmann TJ, Nabhan C, Parikh SA, Hanson CA, et al. Prevalence and characteristics of central nervous system involvement by chronic lymphocytic leukemia. Haematologica 2016;101(4):458-465.

3. Nakanishi T, Ito T, Fujita S, Satake A, Konishi A, Hotta M, et al. Refractory chronic lymphocytic leukemia with central nervous system involvement: a case report with literature review. J Blood Med 2020;11:487-502.

4. Abdulrahman A, Wajda A, Peyman S, Lauren M, Seema N, Abdulrazag A. Central nervous system lymphoma in a patient with chronic lymphocytic leukemia: a case report and literature review. Cureus 2018;10(11):e3660.

5. Mihaljevic B, Smiljanic M, Antic D, Kurtovic ND, Balint MT. Chronic lymphocytic leukemia involvement of central nervous system: clinical diversity, diagnostic algorithm and therapeutic challenges. Ann Indian Acad Neurol 2018;21(1):85-87.

6. Timmers NKLM, de Maar JS, van Kruijsdujk RCM, Klein SK. Central nervous system localisation of chronic lymphocytic leukaemia, description of two very distinct cases and a review of the literature. Ann Hematol 2018;97:1627-1632.

7. Wanquet A, Birsen R, Bonnet C, Boubaya M, Choquet S, Dupuis J, et al. Management of central nervous system involvement in chronic lymphocytic leukaemia: a retrospective cohort of 30 patients. Br J Haematol 2017;176(1):37-49.

8. Hna JH, Wilber ST. Altered mental status in older emergency department patients. Clin Geriatr Med 2013;29(1):101-136.

9. Taylor JW, Flanagan EP, O'Neil BP, Siegal T, Omuro A, DeAngelis L, et al. Primary leptomeningeal lymphoma. Neurology 2013;81:1690-1696.

10. Pinto AL, Ferreira G, Carda JP, Gomes M, Ribeiro ML. Isolated Ritcher's syndrome of the brain: diagnosis in the eye of the beholder. Ann Hematol 2018 ;97(8):1509-1511. 